\title{
Proving Memory Safety of Floating-Point Computations by Combining Static and Dynamic Program Analysis
}

\author{
Patrice Godefroid \\ Microsoft Research \\ Redmond, WA USA \\ pg@microsoft.com
}

\author{
Johannes Kinder \\ Technische Universität Darmstadt \\ Darmstadt, Germany \\ kinder@cs.tu-darmstadt.de
}

\begin{abstract}
Whitebox fuzzing is a novel form of security testing based on dynamic symbolic execution and constraint solving. Over the last couple of years, whitebox fuzzers have found many new security vulnerabilities (buffer overflows) in Windows and Linux applications, including codecs, image viewers and media players. Those types of applications tend to use floating-point instructions available on modern processors, yet existing whitebox fuzzers and SMT constraint solvers do not handle floating-point arithmetic. Are there new security vulnerabilities lurking in floating-point code?

A naive solution would be to extend symbolic execution to floating-point (FP) instructions (months of work), extend SMT solvers to reason about FP constraints (months of work or more), and then face more complex constraints and an even worse path explosion problem. Instead, we propose an alternative approach, based on the rough intuition that FP code should only perform memory safe data-processing of the "payload" of an image or video file, while the non-FP part of the application should deal with buffer allocations and memory address computations, with only the latter being prone to buffer overflows and other security critical bugs. Our approach combines (1) a lightweight local path-insensitive "may" static analysis of FP instructions with (2) a high-precision wholeprogram path-sensitive "must" dynamic analysis of non-FP instructions. The aim of this combination is to prove memory safety of the FP part of each execution and a form of non-interference between the FP part and the non-FP part with respect to memory address computations.

We have implemented our approach using two existing tools for, respectively, static and dynamic x 86 binary analysis. We present preliminary results of experiments with standard JPEG, GIF and ANI Windows parsers. For a given test suite of diverse input files, our mixed static/dynamic analysis is able to prove memory safety of FP code in those parsers for a small upfront static analysis cost and a marginal runtime expense compared to regular dynamic symbolic execution.
\end{abstract}

${ }^{*}$ The work of this author was done mostly while visiting Microsoft.

Permission to make digital or hard copies of all or part of this work for personal or classroom use is granted without fee provided that copies are not made or distributed for profit or commercial advantage and that copies bear this notice and the full citation on the first page. To copy otherwise, to republish, to post on servers or to redistribute to lists, requires prior specific permission and/or a fee.

ISSTA'10, July 12-16, 2010, Trento, Italy.

Copyright 2010 ACM 978-1-60558-823-0/10/07 ...\$10.00.

\section{Categories and Subject Descriptors}

D.2.4 [Software Engineering]: Software/Program Verification; D.2.5 [Software Engineering]: Testing and Debugging; F.3.1 [Logics and Meanings of Programs]: Specifying and Verifying and Reasoning about Programs

\section{General Terms}

Verification, Algorithms, Reliability

\section{Keywords}

Static and Dynamic Program Analysis, Program Verification

\section{INTRODUCTION}

Whitebox fuzzing [10] is a promising new form of security testing based on dynamic test generation $[3,8]$. Starting with a wellformed input, whitebox fuzzing executes the program under test while simultaneously performing symbolic execution to generate input constraints from conditional statements that capture the program execution path. Those constraints are then systematically negated and solved with a constraint solver, generating new test inputs to exercise different execution paths of the program. This process is repeated with the goal of exercising many program paths and finding many bugs. Over the last couple of years, whitebox fuzzers have found many new security vulnerabilities (buffer overflows) in Windows [10] and Linux [14] applications, including codecs, image viewers and media players. Notably, whitebox fuzzing was recently credited to have found roughly one third of all the bugs discovered by file-fuzzing during the development of Microsoft's Windows 7 [7].

Many image and video codecs use floating-point instructions available on modern processors. Yet existing whitebox fuzzers do not generate constraints for floating-point code, perhaps because current mainstream SMT solvers (such as STP, Yices, and Z3, to name a few) do not handle floating-point arithmetic.

In this paper, we present a new technique for proving memory safety of floating-point (FP) computations that does not require precise symbolic reasoning about FP code. The basic idea is to treat all FP values as a single special symbolic value "FP-tag" during symbolic execution, and to leverage existing symbolic evaluation rules to perform a dynamic taint-flow analysis of FP-tags. If an FPtag is ever used to compute a memory address dereferenced during dynamic symbolic execution, an error is generated as a warning.

Our intuition is that such warnings should be rare as floatingpoint computations should be involved only in the processing of the "payload" of an image or video input file, not in buffer allocations and memory address computations, with only the latter being prone to buffer overflows and other security critical bugs. Indeed, 
it would be intuitively surprising if processing, say, a JPEG image of a white flower would result in a memory safe execution, while processing the exact same image but with a red flower would trigger a buffer overflow. A related but simpler phenomenon is data independence in communication protocols [19]: only the control part (i.e., the header and footer) of a packet influences the behavior of a protocol entity, while the data part is just forwarded to a lower or higher protocol layer and is irrelevant to prove correctness. In contrast, image and media formats are usually much more complex, and the distinction between control and data payload is made at runtime by treating differently different parts of the input. For instance, a movie file may start with a declaration that it contains 10,000 chunks of data. After this global header, a stream of all chunks follows, with each having its own private header describing whether it contains audio or video data, its size and encoding. Moreover, assuming we define the payload of a file as the bytes that are processed by FP code, payload data may influence the control flow of the image or movie processor whenever floating-point values are tested in conditional statements.

To prove memory safety of a conditional statement tainted by an FP-tag during symbolic execution, we use a lightweight static analysis for conservatively over-approximating all possible executions of the if-then-else block and then skip the entire block during symbolic execution, provided that statically-computed checks are satisfied at runtime. The satisfiability of those checks guarantees that all possible executions inside the block do not access any memory address computed using FP-tags or input values, and that all their side-effects are conservatively represented by new FP-tags injected when symbolic execution resumes at the end of the block.

We have implemented our approach using two existing tools for static and dynamic x 86 binary analysis, respectively. We present preliminary results of experiments with standard JPEG, GIF and ANI processors embedded in Windows (and deployed on nearly a billion machines worldwide). For a given test suite of diverse input files, our mixed static/dynamic analysis is able to prove memory safety of FP code in the observed executions of those parsers for a small upfront static analysis cost and a marginal runtime expense compared to regular dynamic symbolic execution, hence avoiding costly FP constraint generation and solving, and additional tests to cover FP code.

\section{BACKGROUND: SYSTEMATIC DYNAMIC TEST GENERATION}

Dynamic test generation (see [8] for further details) consists of running the program $P$ under test both concretely, executing the actual program, and symbolically, calculating constraints on values stored in program variables and expressed in terms of input parameters. Side-by-side concrete and symbolic executions are performed using a concrete store $M$ and a symbolic store $S$, which are mappings from memory addresses (where program variables are stored) to concrete and symbolic values, respectively. A symbolic value is any expression $e$ in some theory $\mathcal{T}$ where all free variables are exclusively input parameters. For any program variable $x, M(x)$ denotes the concrete value of $x$ in $M$, while $S(x)$ denotes the symbolic value of $x$ in $S$. For notational convenience, we assume that $S(x)$ is always defined and is simply $M(x)$ by default if no expression in terms of inputs is associated with $x$ in $S$. When $S(x)$ is different from $M(x)$, we say that the program variable $x$ is "symbolic", meaning that its value is a function of some input(s), which is represented by the symbolic expression $S(x)$ associated with $x$ in the symbolic store. We also extend this notation to allow $M(e)$ to denote the concrete value of symbolic expression $e$ when evaluated with the concrete store $M$. The notation + for mappings denotes updating; for example, $M^{\prime}=M+[m \mapsto v]$ is the same map as $M$, except that $M^{\prime}(m)=v$.

The program $P$ manipulates the memory (concrete and symbolic stores) through statements or commands, which are abstractions of the machine instructions actually executed. A command can be an assignment of the form $v:=e$ (where $v$ is a program variable and $e$ is an expression) or $\left[e_{1}\right]:=e_{2}$ (where $\left[e_{1}\right]$ is a memory address dereference at the address defined by evaluating expression $e_{1}$, and $e_{2}$ is an expression), a conditional statement of the form if $e$ then $C^{\prime}$ else $C^{\prime \prime}$ (where $e$ denotes a Boolean expression, and $C^{\prime}$ and $C^{\prime \prime}$ denote the unique ${ }^{1}$ next command to be evaluated when $e$ holds or does not hold, respectively), or stop (which corresponds to a program error or normal termination).

Given an input vector $I$ assigning a value $I_{i}$ to the i-th input parameter, the evaluation of a program defines a unique finite ${ }^{2}$ program execution $s_{0} \stackrel{C_{1}}{\rightarrow} s_{1} \ldots \stackrel{C_{n}}{\rightarrow} s_{n}$ that executes the finite sequence $C_{1} \ldots C_{n}$ of commands and goes through the finite sequence $s_{1} \ldots s_{n}$ of program states. Each program state is a tuple $\langle C, M, S, p c\rangle$ where $C$ is the next command to be evaluated, and $p c$ is a special metavariable that represents the current path constraint. For a finite sequence $w$ of commands (i.e., a control path $w$ ), a path constraint $p c_{w}$ is a formula of theory $\mathcal{T}$ that characterizes the input assignments for which the program executes along $w$. To simplify the presentation, we assume that all the program variables have some unique initial concrete value in the initial concrete store $M_{0}$, and that the initial symbolic store $S_{0}$ identifies the program variables $v$ whose values are program inputs (for all those, we have $S_{0}(v)=I_{i}$ where $I_{i}$ is the corresponding input parameter). Initially, $p c$ is defined to true.

Systematic dynamic test generation [8] consists of systematically exploring all feasible program paths of the program under test by using path constraints and a constraint solver. By construction, a path constraint represents conditions on inputs that need to be satisfied for the current program path to be executed. Given a program state $\langle C, M, S, p c\rangle$ and a constraint solver for theory $\mathcal{T}$, if $C$ is a conditional statement of the form if $e$ then $C^{\prime}$ else $C^{\prime \prime}$, any satisfying assignment to the formula $p c \wedge e$ (respectively $p c \wedge \neg e$ ) defines program inputs that will lead the program to execute the then (respectively else) branch of the conditional statement. By systematically repeating this process, such a directed search can enumerate all possible path constraints and eventually execute all feasible program paths.

The search is exhaustive provided that the generation of the path constraint (including the underlying symbolic execution) and the constraint solver for the given theory $\mathcal{T}$ are both sound and complete, that is, for all program paths $w$, the constraint solver returns a satisfying assignment for the path constraint $p c_{w}$ if and only if the path is feasible (i.e., there exists some input assignment leading to its execution). In this case, in addition to finding errors such as the reachability of bad program statements (like assert (false)), a directed search can also prove their absence, and therefore obtain a form of program verification.

THEOREM 1. (adapted from [8]) Given a program $P$ as defined above, a directed search using a path constraint generation and a constraint solver that are both sound and complete exercises all feasible program paths exactly once.

In this case, if a program statement has not been executed when the

\footnotetext{
${ }^{1}$ We assume program executions are sequential and deterministic.

${ }^{2} \mathrm{We}$ assume program executions terminate. In practice, a timeout prevents non-terminating program executions and issues a runtime error.
} 


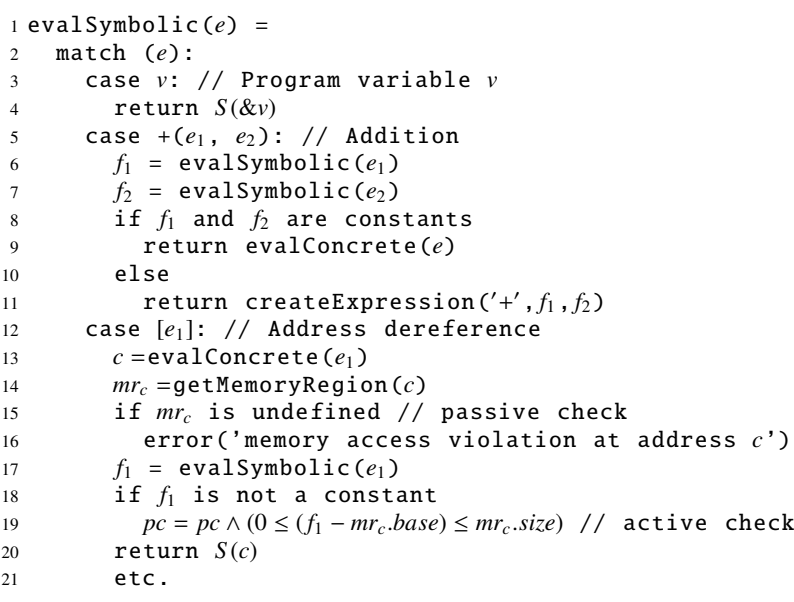

Figure 1: Symbolic expression evaluation.

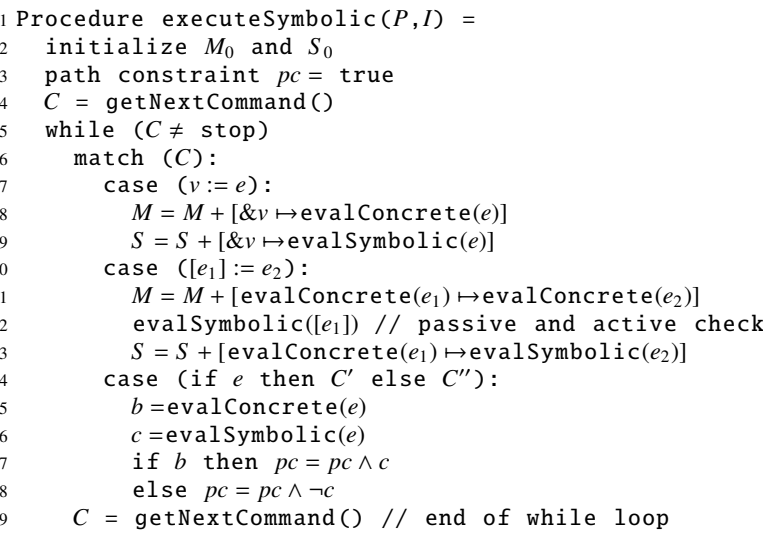

Figure 2: Symbolic execution.

search is over, this statement is not executable in any context. In practice, path constraint generation and constraint solving are usually not sound and complete. When a program expression cannot be expressed in the given theory $\mathcal{T}$ decided by the constraint solver, it can be simplified using concrete values of sub-expressions, or replaced by the concrete value of the entire expression.

Note that the above formalization and theorem do apply to programs containing loops or recursion, as long as all program executions terminate. However, in the presence of a single loop whose number of iterations depends on some unbounded input, the number of feasible program paths becomes infinite. In practice, termination can always be forced by bounding input values, loop iterations, or recursion, at the cost of potentially missing bugs.

Figure 1 illustrates how to symbolically evaluate expressions $e$ involved in program instructions; Figure 2 shows how to generate a path constraint while symbolically executing a program. \&v denotes the address at which the value of $v$ is stored. As in [9], all symbolic expressions $e$ ever used in the left hand-side (lines 9 and 13 of Figure 2) or right hand-side (line 12 of Figure 2) of an assignment statement or in the Boolean expression of a conditional statement (line 16 of Figure 2) are checked for memory access violations. Whenever a memory address is dereferenced during execution, an expression of the form $[e]$ is evaluated to compute that address. The concrete value $c$ of the address is checked "passively" (in line 15 of Figure 1) to make sure it points to a valid memory region $m r_{c}$; then the symbolic expression $e$ is also checked "actively" by injecting a new constraint in the path constraint (line 19 of Figure 1) to make sure other input values cannot trigger a buffer overflow or underflow at this point of the program execution [9]. How to keep track of the base address $m r_{c}$.base and size $m r_{c}$. size of each valid memory region $m r_{c}$ during the execution of the program $P$ is discussed in [5]. To simplify the presentation, Figure 1 only handles single symbolic pointer dereferences (by returning the value $S(c)$ in line 20), but could be extended to handle multiple levels of pointer indirections [5].

Given two program executions $w$ and $w^{\prime}$, we write $w \equiv_{P} w^{\prime}$ if they execute the same (finite) sequence of commands. Observe that $w \equiv_{P} w^{\prime}$ implies $p c_{w}=p c_{w^{\prime}}$ since both execute the same control path. Let an extended path constraint epc denote a path constraint extended with buffer-overflow checks injected as in line 19 of Figure 1. We write $w \equiv_{P+B} w^{\prime}$ if $w \equiv_{P} w^{\prime}$ and $e p c_{w}=e p c_{w^{\prime}}$. Thus, two executions $w$ and $w^{\prime}$ are equivalent with respect to $\equiv_{P+B}$ if they execute the same control path in the extended program $P+B$ which extends the original program $P$ with bound checks for all memory accesses. Such bound checks are useful to prove memory safety.

THEOREM 2. (adapted from [9]) Given an extended program $P+$ $B$ as defined above, a directed search using an extended path constraint generation and a constraint solver that are both sound and complete exercises all feasible program paths exactly once. Moreover, if no runtime error is ever generated by line 16 of Figure 1, all program executions are memory safe.

\section{PROBLEM DEFINITION}

For security analysis, the advantages of binary analyses are well established: static $[2,12]$ or dynamic $[10,14]$ binary analyses allow to analyze the exact program that is being shipped to customers and that includes important details such as code transformations performed by compilers. In this work, we adopt a similar approach and build upon two existing tools for, respectively, static and dynamic x86 binary analysis of Windows applications. Specifically, the tool we extend for dynamic symbolic execution handles a large set of x86 instructions, but no floating-point (FP) instructions.

We use the term FP instructions to refer to the about 100 floating point and over 300 SIMD instructions in the $x 86$ architecture [11]. Floating point instructions execute on the $\mathrm{x} 87 \mathrm{FPU}$, which used to be a separate physical unit on early x 86 platforms. They primarily read from and write to the FPU's own register stack (st ( $(\theta)$ to st (7)) and its status-, control-, and tag-registers. SIMD (Single Instruction, Multiple Data) instructions have been introduced into $\mathrm{x} 86$ as the MMX, 3DNow!, SSE, SSE2, SSE3, SSSE3, and SSE4 extensions [17]. These instructions also use special registers (mmx0-7 as aliases of the FPU register stack, xmm0-15, and the control- and status register) and are specifically tailored towards fast vector operations. We refer to all registers used exclusively by floating point and SIMD instructions as FP-registers. Thus, the value stored in an FP-register can only be moved to memory or a regular register by an FP instruction.

For instance, here is an example of floating point code that loads a variable, multiplies it with a constant, and stores the result:

$$
\mathrm{x}:=\mathrm{x} * 2.3 ; \quad \begin{aligned}
& \text { fld qword ptr } \mathrm{x} \\
& \text { fmul qword ptr flt2_3 } \\
& \text { fstp qword ptr } \mathrm{x}
\end{aligned}
$$

Formally, we now assume that the program $P$ to be analyzed actually executes two types of assignment statements: (1) floatingpoint $(F P)$ assignments of the form $v:=e$ or $\left[e^{\prime}\right]:=e$ where $v$ 


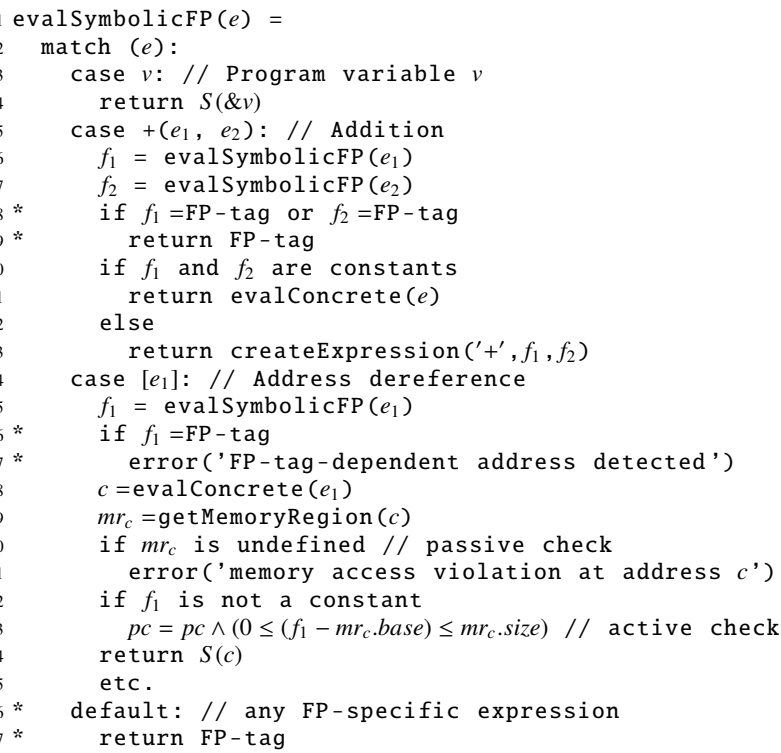

Figure 4: New symbolic expression evaluation with FP-tag.

regular symbolic execution of the assignment $C$ is replaced ${ }^{3}$ as follows: the symbolic value of every memory address and register in WriteAddrOrReg $(C)$ becomes the special symbolic value $F P$-tag in the symbolic store (line 3 ).

FP-tag is a single symbolic value that represents all possible floating-point (concrete) values during symbolic execution, as well as all symbolic expressions that depend on an FP value, hence including all possible symbolic expressions in theory $\mathcal{T}^{\prime} \backslash \mathcal{T}$. Symbolic expression evaluation is easily extended to handle FP-tags as illustrated in Figure 4. The new value FP-tag is an "absorbing element" with respect to symbolic expression evaluation: the value of any expression containing FP-tag is FP-tag (e.g., see lines 8-9 of Figure 4). Also, any FP-specific expression (i.e., in $\mathcal{T}^{\prime} \backslash \mathcal{T}$ ) returns FP-tag (lines 26-27 of Figure 4).

As in regular symbolic execution, all memory address dereferences are checked both passively and actively for memory access violations (see Section 2). If any memory address ever depends on an FP-tag, an error is (conservatively) generated in line 17 of Figure 4.

The algorithm of Figure 3 also generates an error if the Boolean expression of any conditional statement ever becomes the value FPtag (lines 24-25).

Let $M(m, s)$ and $S(m, s)$ denote the value of memory location $m$ in state $s$ in the concrete store $M$ or symbolic store $S$, respectively. Given any two states $s$ and $s^{\prime}$, we write $s \equiv_{F P} s^{\prime}$ if $\forall m: M(m, s)=$ $M\left(m, s^{\prime}\right)$ or $S(m, s)=S\left(m, s^{\prime}\right)=$ FP-tag. Thus, the $\equiv_{F P}$ equivalence is very tight: two states that are $\equiv_{F P}$ equivalent can only differ in any memory location by the concrete floating-point value abstracted by the symbolic value FP-tag.

We extend the notation to define equivalence classes of program executions: given two program executions $w=s_{0} \stackrel{C_{1}}{\rightarrow} s_{1} \ldots \stackrel{C_{n}}{\rightarrow} s_{n}$ and $w^{\prime}=s_{0}^{\prime} \stackrel{C_{1}^{\prime}}{\rightarrow} s_{1}^{\prime} \ldots \stackrel{C_{n}^{\prime}}{\rightarrow} s_{n}^{\prime}$, we write $w \equiv_{F P} w^{\prime}$ if $\forall i: s_{i} \equiv_{F P}$ $s_{i}^{\prime} \wedge C_{i}=C_{i}^{\prime}$.

\footnotetext{
${ }^{3}$ To keep the notation simple, in case of FP assignments, we assume the symbolic store updates in lines 14 and 19 of Figure 3 are no-ops (e.g., when $v$ is a FP variable) that are subsumed by the updates in line 3 of checkAssignment ().
}

THEOREM 3 (MEMORY SAFETY). Given a program $P$ as defined above with FP and non-FP assignments, if the algorithm of Figures 3 and 4 does not generate any error for an execution $w$, then for all executions $w^{\prime}$ such that $w^{\prime} \equiv_{F P} w$, we have $w^{\prime} \equiv_{P+B} w$, and all executions $w^{\prime}$ are memory safe.

Proof. We first show that if the algorithm does not generate any error for execution $w$, then $w \equiv_{F P} w^{\prime}$ implies $w \equiv_{P+B} w^{\prime}$. First, during the execution $w$, no conditional statement ever depends on an FP-tag (otherwise contradiction by lines $24-25$ of Figure 3), therefore $w \equiv_{P} w^{\prime}$. Second, all memory accesses during the execution $w$ are checked in lines 14-24 of Figure 4 (due to calls to evalSymbolicFP() in lines 14, 18, 19 and 23 of Figure 3); moreover, no memory address ever depends on an FP-tag (otherwise an error would be generated in line 17 of evalSymbolicFP()). This implies $w \equiv_{P+B} w^{\prime}$.

Since $w$ is memory safe (any non-memory safe access would trigger an error by line 21 of evalSymbolicFP()), $w \equiv_{P+B} w^{\prime}$ implies that $w^{\prime}$ is memory safe, too.

COROLLARY 4. If the number of program executions $w$ that are distinct with respect to $\equiv_{P+B}$ is finite, all those can be enumerated by a directed search and, if the algorithm of Figures 3 and 4 never generates any error for any of these executions, the entire program $P$ is memory safe.

Note that if the algorithm of Figures 3 and 4 reports an error of the type "FP-tag-dependent address or test detected", this does not necessarily imply that the program is not memory safe. Indeed, the injection of FP-tags in line 3 of checkAssignment () and their propagation using rules like in lines 8-9 of evalSymbolicFP() are conservative: the FP-tag value abstracts and blends together concrete values that might have been otherwise distinguished by FP constraints injected in the path constraint either in lines 26-27 of executeSymbolicFP() or in line 23 of evalSymbolicFP(), if symbolic execution and constraint solving had been extended to handle FP constraints in $\mathcal{T}^{\prime}$.

\section{DEALING WITH FP-DEPENDENT CONDITIONAL STATEMENTS}

The previous algorithm reports an error whenever the Boolean expression $e$ of any conditional statement depends on an FP-tag. Unfortunately, FP-dependent conditional statements that test floating-point values are frequent in practice, as illustrated in the second example in Section 3 and as will be discussed later in the experiments of Section 7. In those cases, the previous algorithm is not sufficient, as it will generate too many alarms.

In this section, we present a refined algorithm that can eliminate many of those alarms. The main idea is to treat an entire if-thenelse block depending on an FP-tag as a single FP instruction: a lightweight static analysis is used to over-approximate all possible executions inside that block, starting from the conditional statement $C$ until its unique immediate postdominator instruction ipdom $(C)$, and to compute two sets $\operatorname{AddrReg}(C)$ and WriteAddrOrReg $(C)$ for the entire block starting at $C$ :

- $\operatorname{AddrReg}(C)$ is a set of regular (non-FP) registers whose value at the time of executing $C$ may be used to compute a memory address being read or written during the execution of $C$ to ipdom $(C)$; if any value from memory may be used to compute an address (multiple dereferences) during the execution of $C$ to ipdom $(C), \operatorname{AddrReg}(C)$ is set to a special value "unsafe". 


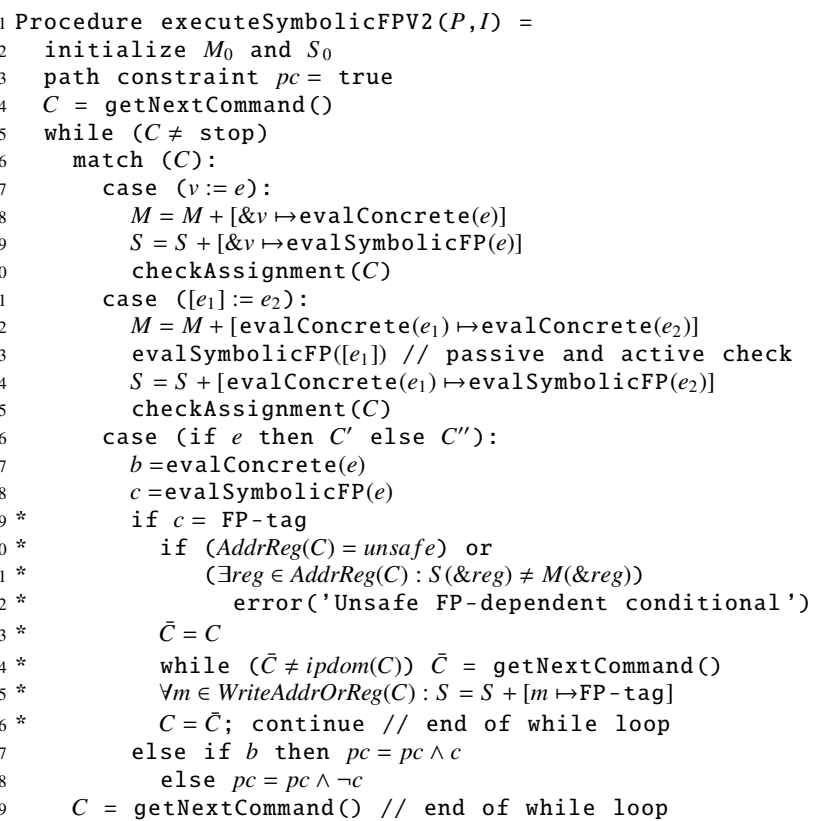

Figure 5: New symbolic execution extended to FP assignments and FP-dependent conditionals.

- WriteAddrOrReg $(C)$ is a set of memory addresses or regular (non-FP) registers that may be written to during the execution of $C$ to ipdom $(C)$; if a memory address cannot be expressed by the static analysis for the time immediately before executing ipdom $(C)$ (e.g., a pointer register is overwritten after an access), $\operatorname{AddrReg}(C)$ is set to "unsafe".

The immediate postdominator ipdom $(C)$ can be efficiently determined using standard algorithms [13]; the two sets $\operatorname{AddrReg}(C)$ and WriteAddrOrReg $(C)$ are over-approximated and a default value of "unsafe" is used for $\operatorname{AddrReg}(C)$ when complicated code is encountered. There are different ways to cheaply compute this overapproximation. In Section 6 we outline a simple algorithm that yields sufficiently precise approximations without full-blown symbolic execution. Before executing the program, this static analysis algorithm is run to pre-compute $\operatorname{ipdom}(C), \operatorname{AddrReg}(C)$ and WriteAddrOrReg $(C)$ for every conditional statement $C$ in the program under test.

Figure 5 presents the refined symbolic execution algorithm which is similar to the one of Figure 3 except for the new lines 19-26 prefixed with a *. Now, whenever symbolic execution hits an FPdependent conditional statement $C$, instead of immediately reporting an error, the refined algorithm attempts to proceed by "skipping" the entire if-then-else block. First, if any register in the statically precomputed $\operatorname{AddrReg}(C)$ has any symbolic value, i.e., is either input-dependent or FP-tag, an error is generated (lines 20-22). Otherwise, symbolic execution proceeds until ipdom $(C)$ is reached (lines 23-24). Just before resuming symbolic execution at ipdom $(C)$ (line 26), the symbolic value of every memory address and register in WriteAddrOrReg $(C)$ becomes FP-tag in the symbolic store (line 25).

Given two program executions $w$ and $w^{\prime}$, we write $w \equiv_{P+F P} w^{\prime}$ if $w \equiv_{F P} w^{\prime}$ except at FP-dependent conditional statements $C$, where they are allowed to follow different branches and execute different commands until either both of them eventually reach ipdom $(C)$, or at least one of them never reaches $\operatorname{ipdom}(C)$ (due to early termina- tion or non-termination). Thus, the equivalence relation $\equiv_{P+F P}$ is weaker than $\equiv_{P}$ and $\equiv_{F P}$. We also write $w \equiv_{P+F P+B} w^{\prime}$ if $w \equiv_{P^{\prime}+F P}$ $w^{\prime}$ where $P^{\prime}$ is $P$ extended with bound checks for all memory accesses.

A program execution $w$ is called attacker memory safe if every buffer access during $w$ in the program $P$ extended with bound checks for all memory accesses is either within bounds (i.e., is memory safe) or is input-independent, i.e., its address has no inputdependent symbolic value, and hence is not directly controllable by an attacker through the untrusted input interface.

Thus, the notion of "attacker memory safe" is weaker than memory safety: if a program execution is memory safe, this implies that the execution is attacker memory safe, while the converse does not hold. Nevertheless, in the context of security testing, we are primarily interested in attacker memory safety since buffer overflows where the overflow is fixed and cannot be directly controlled by the attacker are likely much harder to exploit. We now show that the algorithm of Figure 5 can only guarantee this weaker form of memory safety.

Theorem 5 (ATTACKeR MEMORY SAFETY). Given a program $P$ as defined above with FP and non-FP assignments and regular and FP-dependent conditional statements, if the algorithm of Figures 5 and 4 does not generate any error for an execution $w$, then for all executions $w^{\prime}$ such that $w^{\prime} \equiv_{F P} w$, we have $w^{\prime} \equiv_{P+F P+B} w$, and all executions $w^{\prime}$ are attacker memory safe.

Proof. We show that if the algorithm does not generate any errors for execution $w$, then $w \equiv_{F P} w^{\prime}$ implies $w \equiv_{P+F P+B} w^{\prime}$. Since $w \equiv_{F P} w^{\prime}$, for each conditional statement $C$ reached by those executions, either the conditional statement does not depend on an FP-tag and the two executions execute the same branch at $C$ and subsequent instructions, or the conditional statement depends on an FP-tag and the two executions are allowed to diverge until ipdom $(C)$ is reached. Moreover, all possible side-effects of all possible executions between $C$ and $\operatorname{ipdom}(C)$ are covered with FPtags when $\operatorname{ipdom}(C)$ is executed (line 25 of executeSymbolicFPV2()). Thus, if $s$ and $s^{\prime}$ denote the states of $w$ and $w^{\prime}$ when they both reach ipdom $(C)$, respectively, we have $s \equiv_{F P} s^{\prime}$. This implies that $w \equiv_{P+F P} w^{\prime}$.

During the execution of $w$, all memory accesses performed during symbolic execution are checked to be memory safe in lines 14-24 of evalSymbolicFP() (called in lines 9, 13, 14 and 18 of executeSymbolicFPV2()). Moreover, all memory accesses that can possibly happen during all possible program executions between every FP-conditional statement $C$ and $\operatorname{ipdom}(C)$ are conservatively represented by $\operatorname{AddrReg}(C)$, and all those memory accesses are checked in lines 20-21 to be both input- (i.e., attacker-) independent and FP-tag-independent (otherwise an error would be generated in line 22). Therefore, no memory access ever depends on an FP-tag and we have $w \equiv_{P+F P+B} w^{\prime}$.

Furthermore, all memory accesses in $w$ are either memory safe during symbolic execution or input-independent between FP-dependent conditional statements $C$ and their $\operatorname{ipdom}(C)$. By definition, $w$ is then attacker memory safe. Since $w \equiv_{P+F P+B} w^{\prime}, w^{\prime}$ is attacker memory safe too.

The correctness guarantees provided by Theorem 5 are weaker than those provided by Theorem 3 , since attacker memory safety is weaker than memory safety. Indeed, executions $w^{\prime}$ such that $w \equiv_{F P} w^{\prime}$ can take different executions paths between FP-dependent conditional statements $C$ and their $\operatorname{ipdom}(C)$, and some of those other paths may trigger memory access violations or other runtime errors (such as division-by-zero or infinite loops). Since we do not generate FP constraints for those conditional statements 
$C$, we cannot generate tests to exercise those paths and hit those errors. Nevertheless, our algorithm can guarantee that no such path ever contains an input or FP-dependent memory access.

\section{PROTOTYPE IMPLEMENTATION}

We have implemented our approach as an extension to the existing whitebox fuzz testing tool SAGE [10]. For exploring execution paths of a program under test, the tool starts from a well-formed input file and systematically manipulates input bytes to drive execution into new branches.

We extended the symbolic execution engine of SAGE to incorporate our new algorithm in Figure 5. To calculate the AddrReg and WriteAddrOrReg sets, we built a separate static analysis tool that processes the binary under test and all referenced dynamic libraries. The static analysis tool is based on Microsoft Vulcan [4], a mature framework for parsing, analyzing, and instrumenting compiled binaries. Dynamic libraries (DLLs) can be conditionally loaded at runtime, and it is not always possible to statically determine the full set of DLLs that may be accessed. If symbolic execution encounters an instruction belonging to the address space of a library for that no static information has been generated, the static analysis has to be invoked on that particular library to generate the missing information.

\section{Static Information.}

For an executable or DLL, our tool computes the AddrReg and WriteAddrOrReg sets for all individual FP instructions and for all conditional jumps. We tried to keep the static analysis as fast and simple as possible but as precise as necessary to prove FP dependent conditional jumps to be attacker memory safe (provided that the values of registers in AddrReg are input-independent and not FP-tag). Without knowledge about the actual semantics of an instruction, disassemblers such as the one included in Vulcan are still able to extract source and destination operands from it by decoding the relevant bytes in the instruction stream. Typically, elaborate tables are used for mapping bytes to instructions, which also expose implicit register operands (e.g., eax is an implicit source and target in mul cx). When processing an individual FP instruction, target operands are added to the WriteAddrOrReg set.

The WriteAddrOrReg and AddrReg sets for conditionals need to capture the effects of all control-dependent instructions. We have implemented a flow insensitive interprocedural analysis for determining both sets. Both branches of each conditional jump are explored up to but not including the immediate postdominator of the jump. For each instruction in the branches, its destination operands are added to WriteAddrOrReg, and the base and index registers of any memory operands are added to AddrReg. If the intersection of both sets is non-empty, i.e., if any of the dereferenced registers is manipulated in the control-dependent block, our analysis reports the conditional as unsafe. For example, consider the following loop controlled by an FP expression:

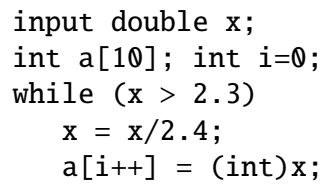

Our static analysis would return "unsafe" for the condition controlling the loop when analyzing it up to its immediate postdominator (which is the next statement after the while loop). This is necessary, since pointer manipulation in an FP-dependent control block could be vulnerable to an attack that might not be detected by the dynamic analysis.
When all pointers are constant within a conditional block, all target memory addresses can be easily determined and represented in a single pass. No special treatment of loops is required, since the analysis is flow and path insensitive. In our implementation, memory locations in WriteAddrOrReg can be of the form [base + offset], where base is a register and offset an integer. The restriction that pointers remain constant also excludes multiple dereferences within a single block, which in $\mathrm{x} 86$ requires assigning an address to an intermediate register.

Disallowing any changes to dereferenced registers is too strict for handling the stack pointer (esp) and push/pop. E.g., parameters of function calls are pushed onto the stack in Windows' standard calling convention, thereby modifying and dereferencing the stack pointer in one instruction. We use a standard path-insensitive stack-height analysis supplied by Vulcan to determine at every instruction the offset of esp from the base of the procedure's stack frame. In compiler generated code, this offset is always constant for a certain instruction. We can thus replace references to esp-based local variables with equivalent stack frame based memory locations in the WriteAddrOrReg set. At the immediate postdominator of the conditional jump, these locations are then translated back to esp based addresses.

Function calls within a conditional block are supported by our tool, which recursively calculates AddrReg and WriteAddrOrReg for complete procedures in standard bottom up fashion and adds these sets at call sites. Care must be taken to correctly translate stack frame relative addresses in the WriteAddrOrReg set of the callee into the stack frame of the caller. Some procedures set up and use a frame pointer in ebp for referencing local variables, so ebp would show up in both sets. We solve this problem by using the same translation as for esp, and express all ebp based local variables as stack frame relative addresses (in Visual C, ebp always has an offset of 4 from the stack frame).

This approach also handles recursive or mutually recursive functions, since it is not necessary for WriteAddrOrReg to record updates to stack frames which have become invalid at the immediate postdominator of the original conditional statement. Any other side effects are over-approximated by one pass of a function, since other pointers than the stack and frame pointer have to remain constant, otherwise the static analysis is aborted and returns the default value unsafe for AddrReg.

\section{Control Flow Information.}

Calculating immediate postdominators for conditional jumps requires precise control flow information. Our prototype correctly handles procedures with multiple exits, non-returning procedure calls (e.g., ExitProcess), inter-procedure jumps (e.g., to shared error handling code), and the tail-jump optimization; it currently does not support implicit control flow through exceptions raised by instructions or in callees. If an immediate postdominator cannot be statically determined for a conditional jump (e.g., because one branch terminates), the implementation reports the conditional to be unsafe.

We rely on Vulcan to decode instructions, identify procedures, and provide control flow information. Soundness of our implementation therefore depends on the soundness of Vulcan's analysis.

\section{SSA optimizations in the C runtime library.}

With our assumption that floating point and SSE instructions are used exclusively for payload processing, we did not expect pointers to be modified by them. However, it turned out that the implementations of memset and memcpy in Microsoft's C runtime use SSA 
instructions for speed, if the CPU supports them and if the memory blocks happen to be 16-byte aligned.

Both cases lead to memory locations falsely being tagged as floating point data (set to FP-tag), including pointers inside larger structures. For memset, we extended the static analysis to understand the PXOR (16-byte XOR) and MOVDQA (16-byte move) instructions and to perform a simple intraprocedural constant propagation. This suffices to identify the SSA move instructions inside memset as assignments of a constant integer value, for which WriteAddrOrReg is empty. For memcpy, the symbolic execution should be aware that memory areas are being copied, since whenever a portion of input-dependent data is copied, its symbolic value should be preserved. We handle this as a special case during symbolic execution of the specific code sequence of memcpy to keep track of the size, source and target addresses of the memory to be copied.

\section{EXPERIMENTAL RESULTS}

We report in this section detailed results of experiments with our implementation and test drivers that invoke the built-in JPEG, GIF, and ANI image parsers embedded in Windows Vista. Those parsers are implemented in code spread across various Windows DLLs. For each parser, we first preprocessed all required libraries using our static analysis tool. Many of the libraries are unrelated to the actual parsing but are used for purposes like file access or setting up the Microsoft COM services. This foundation of common library code is share by all parsers, so there is a rather large overlap in the static information used for them.

\section{Static Preprocessing.}

Table 1 gives a breakdown of the results for the static analysis phase, both individually for each DLL and as a total for each parser. We analyzed 20 DLLs in total; the JPEG parser loaded 16, the one for GIF 19, and the one for ANI 15 DLLs. Processing all required DLLs per parser took about 5 to $10 \mathrm{~min}$ on a regular desktop machine. One of the DLLs, shell32.dll, unfortunately was protected by binary obfuscation and could not be processed by our prototype.

Our tool created the static information for all conditional jumps in the program, not only for those which are FP dependent at runtime, since it is not generally possible to statically determine the set of FP-dependent conditionals. For over $80 \%$ of the conditional jumps, our lightweight static analysis is not precise enough to prove anything, and has to flag the conditional as unsafe. However, we do not try to statically prove memory safety of arbitrary code, but are only interested in those conditional statements which become FP-dependent at runtime. All other conditionals should eventually be explored by the algorithm for directed testing and are thus already covered. We designed the static analysis towards the patterns we saw for conditional jumps inside floating point program logic, extending its power as required to cover all or almost all cases. In particular, our static analysis reports as unsafe a conditional statement if in one of the branches the same pointer is both modified and dereferenced (with the exception of the stack and frame pointers, as explained in the previous section). This includes multiple pointer dereferences (e.g., expressions such as **p), loops over arrays, and long code sequences where the same register is used for different pointers. FP-dependent conditionals in the file parsers we looked at are usually relatively short and/or limit their effects to FP registers, so we were able to avoid implementing a more expensive analysis. We did, however, have to make our analysis interprocedural, as some of the FP-dependent conditionals contained function calls.

About $6 \%$ of all conditional jumps are determined to be unconditionally memory safe, which is the case if the conditional block contains no dereferences in either branch and AddrReg is empty. The remaining conditionals do contain dereferences and are conditionally safe: their AddrReg is nonempty and the registers it contains need to be checked for FP-dependent values during symbolic execution.

\section{Symbolic Execution.}

In designing our experiments, we were interested in checking whether the missing floating point support could have caused our existing whitebox fuzzer to miss bugs. If our assumption that FP instructions do not interfere with security critical code is correct, ignoring FP instructions did not miss any security bugs. We therefore focused on replaying the symbolic execution of interesting input files to determine whether all executions terminate without issuing warnings, which could be raised due to unsafe FP-dependent conditionals or FP-dependent memory accesses.

We ran SAGE's symbolic execution on diverse seed files and disabled constraint solving, so that for each seed file we observed one complete symbolic execution trace. We used twelve different seed files per format, randomly selected from a suite of regression tests. The files were of various sizes, to a combined total size of $238 \mathrm{kB}$ in the case of JPEG. Only for JPEG we saw a significant amount of new instructions being covered compared to a single seed file, and in fact some of the seed files caused more DLLs to be loaded by the parser. Still, no new warnings were raised compared to the symbolic execution of the trace for a single seed file per format. Overall, our implementation of symbolic execution with FP tags, processing of static information and associated dynamic checks consistently implies a $20 \%$ runtime overhead compared to symbolic execution without FP tags, and therefore comes at an overall marginal runtime cost.

Table 2 lists the results obtained from symbolically executing the test drivers on one seed file each; as mentioned above, results for the other input files are very similar. The total runtime for the extended symbolic execution were 101 secs for JPEG, 73 secs for GIF, and 5 seconds for ANI. These executions were performed with small seed files of 1,092 bytes for JPEG, 2,957 bytes for GIF, and 2,512 bytes for ANI. Numbers in Table 2 are split between occurrences of instructions (including repeated executions) and unique instructions, and also between instructions in the full trace from initialization to termination and instructions executed after at least one input byte has been read in the execution trace.

For instance, we ran the JPEG test driver and traced 26.7 million instructions (86763 unique), of which 22 million occurred after reading input. It executed only 89 unique FP instructions after the input was read, a large portion of which was part of an inner loop of optimized SSE2 instructions to perform a discrete cosine transform. All three parsers executed FP instructions at some point; in the case of GIF, however, all FP instructions occurred before any input was read. None of the FP instructions and also none of the regular non-branch instructions was found to be unsafe, i.e., no instruction ever dereferenced an FP-dependent value.

All three parsers executed conditional jumps where the EFLAGS register was tagged as being FP-dependent, thus confirming the need for the over-approximating static analysis we introduced in Section 5. Table 2 shows the total number of such FP-dependent conditional jumps, as well as the number of safe and unsafe FP conditionals. An FP-dependent conditional $C$ is listed as safe if the block up to $i p d o m(C)$ was successfully over-approximated and found to be memory safe using $C$ 's static information. $C$ is listed as unsafe if the static analysis has failed and AddrReg in C's static information was set to unsafe, or if AddrReg contains registers holding FP-tagged values at the time of the jump (we never encountered 


\begin{tabular}{|c|c|c|c|c|c|c|c|c|c|c|}
\hline DLL & JPEG & GIF & ANI & All instr. & FP instr. & Conditionals & Safe & Cond. Safe & Unsafe & Time \\
\hline advapi32 & $\checkmark$ & $\checkmark$ & $\checkmark$ & 156442 & 75 & 13370 & $3.6 \%$ & $10.0 \%$ & $86.3 \%$ & $27 \mathrm{~s}$ \\
\hline clbcatq & $\checkmark$ & $\checkmark$ & & 114240 & 100 & 12668 & $24.5 \%$ & $7.8 \%$ & $67.7 \%$ & $27 \mathrm{~s}$ \\
\hline comctl32 & & $\checkmark$ & $\checkmark$ & 376620 & 344 & 31335 & $6.1 \%$ & $14.6 \%$ & $79.3 \%$ & $47 \mathrm{~s}$ \\
\hline gdi32 & $\checkmark$ & $\checkmark$ & $\checkmark$ & 81834 & 366 & 8785 & $3.6 \%$ & $9.9 \%$ & $86.5 \%$ & $11 \mathrm{~s}$ \\
\hline GdiPlus & & $\checkmark$ & & 476642 & 32147 & 42154 & $5.4 \%$ & $13.4 \%$ & $81.3 \%$ & $184 \mathrm{~s}$ \\
\hline imm32 & $\checkmark$ & $\checkmark$ & $\checkmark$ & 26178 & 0 & 2712 & $5.3 \%$ & $4.9 \%$ & $89.9 \%$ & $6 s$ \\
\hline kernel32 & $\checkmark$ & $\checkmark$ & $\checkmark$ & 15958 & 12 & 15958 & $4.1 \%$ & $12.4 \%$ & $83.4 \%$ & $33 \mathrm{~s}$ \\
\hline lpk & $\checkmark$ & $\checkmark$ & $\checkmark$ & 5389 & 45 & 658 & $8.1 \%$ & $16.1 \%$ & $75.8 \%$ & $2 \mathrm{~s}$ \\
\hline msctf & $\checkmark$ & $\checkmark$ & $\checkmark$ & 159228 & 357 & 13985 & $4.5 \%$ & $8.3 \%$ & $87.1 \%$ & $31 \mathrm{~s}$ \\
\hline msvert & $\checkmark$ & $\checkmark$ & $\checkmark$ & 147640 & 5757 & 16260 & $6.0 \%$ & $12.1 \%$ & $81.9 \%$ & $35 \mathrm{~s}$ \\
\hline ntdll & $\checkmark$ & $\checkmark$ & $\checkmark$ & 207815 & 649 & 18876 & $5.1 \%$ & $12.6 \%$ & $82.3 \%$ & $40 \mathrm{~s}$ \\
\hline ole32 & $\checkmark$ & $\checkmark$ & & 367226 & 99 & 32677 & $4.8 \%$ & $7.1 \%$ & $88.1 \%$ & $81 \mathrm{~s}$ \\
\hline oleaut32 & $\checkmark$ & $\checkmark$ & & 148777 & 1335 & 15484 & $7.1 \%$ & $9.6 \%$ & $83.3 \%$ & $25 \mathrm{~s}$ \\
\hline rpcrt4 & $\checkmark$ & $\checkmark$ & $\checkmark$ & 240231 & 57 & 18603 & $5.6 \%$ & $9.5 \%$ & $84.9 \%$ & $31 \mathrm{~s}$ \\
\hline shell32 & & $\checkmark$ & $\checkmark$ & - & - & - & - & - & - & - \\
\hline shlwapi & & $\checkmark$ & $\checkmark$ & 73092 & 0 & 6914 & $7.5 \%$ & $12.3 \%$ & $80.3 \%$ & $9 \mathrm{~s}$ \\
\hline user32 & $\checkmark$ & $\checkmark$ & $\checkmark$ & 121223 & 0 & 11314 & $7.3 \%$ & $12.6 \%$ & $80.0 \%$ & $16 \mathrm{~s}$ \\
\hline usp10 & $\checkmark$ & $\checkmark$ & $\checkmark$ & 79990 & 2 & 8394 & $7.7 \%$ & $11.9 \%$ & $80.5 \%$ & $11 \mathrm{~s}$ \\
\hline uxtheme & $\checkmark$ & $\checkmark$ & $\checkmark$ & 62276 & 110 & 5488 & $5.9 \%$ & $10.9 \%$ & $83.2 \%$ & $7 \mathrm{~s}$ \\
\hline WindowsCodecs & $\checkmark$ & & & 193415 & 6370 & 16926 & $4.4 \%$ & $7.4 \%$ & $88.2 \%$ & $35 \mathrm{~s}$ \\
\hline JPEG (Total) & & & & 2127862 & 15334 & 212158 & $6.4 \%$ & $9.8 \%$ & $83.8 \%$ & $418 \mathrm{~s}$ \\
\hline GIF (Total) & & & & 2860801 & 41455 & 275635 & $6.4 \%$ & $11.1 \%$ & $82.5 \%$ & $623 \mathrm{~s}$ \\
\hline ANI (Total) & & & & 1753916 & 7774 & 172652 & $5.5 \%$ & $11.7 \%$ & $82.8 \%$ & $306 \mathrm{~s}$ \\
\hline
\end{tabular}

Table 1: Results from static analysis of DLLs used by the parsers.

\begin{tabular}{|c|c|c|c|c|c|c|c|c|c|c|c|}
\hline & & \multicolumn{2}{|c|}{ All instructions } & \multicolumn{2}{|c|}{ FP instructions } & \multicolumn{2}{|c|}{ Total FP cond. } & \multicolumn{2}{|c|}{ Safe FP cond. } & \multicolumn{2}{|c|}{ Unsafe FP cond. } \\
\hline & & Full & Input & Full & Input & Full & Input & Full & Input & Full & Input \\
\hline \multirow{2}{*}{ JPEG } & Occurrences & 26712705 & 21983468 & 7826 & 7320 & 45 & 4 & $39(87 \%)$ & 4 & $6(13 \%)$ & 0 \\
\hline & Unique & 86763 & & 104 & 89 & 28 & 1 & $26(93 \%)$ & 1 & $2(7 \%)$ & 0 \\
\hline \multirow{2}{*}{ GIF } & Occurrences & 8952406 & 4786801 & 3856 & 0 & 435 & 0 & $299(69 \%)$ & 0 & $136(31 \%)$ & 0 \\
\hline & Unique & 133958 & & 68 & 0 & 36 & 0 & $32(89 \%)$ & 0 & $4(11 \%)$ & 0 \\
\hline \multirow{2}{*}{ ANI } & Occurrences & 1581268 & 1207886 & 134 & 39 & 41 & 21 & $35(85 \%)$ & 15 & $6(15 \%)$ & 6 \\
\hline & Unique & 29722 & & 16 & 13 & 27 & 7 & $25(93 \%)$ & 5 & $2(7 \%)$ & 2 \\
\hline
\end{tabular}

Table 2: Results from FP-extended symbolic execution for one input file per parser.

the latter case in our experiments). The JPEG trace contained 45 occurrences of FP-dependent conditional jumps, of which 6 (corresponding to 2 unique jumps) had an unsafe precomputed AddrReg and 39 were found to be safe. However, all unsafe conditionals were executed before any data was read from the input file; if the attacker is only able to control the input file, which corresponds to our threat model, it is not possible for him to control the branching behavior of these instructions. Hence, a bug in these conditionals would surface for all or no inputs, and the entire execution is attacker memory safe.

In the GIF trace 4 out of 36 unique FP-dependent conditionals raised warnings, but similar to JPEG, all of them occurred before any input was read.

For ANI, however, we observed two unsafe unique conditional jumps after reading the input file. These were the same two conditional jumps from the same DLL (uxtheme.dll) as in the JPEG trace. Both jumps had an "unsafe" value for AddrReg; the static analysis was unable to deduce memory safety because in both cases one of the branches called a rounding function containing sophisticated error handling code that involves multiple functions for notifying an attached debugger and setting a global error status. How- ever, after a careful visual inspection of the disassembled code, we are confident the function containing both jumps belongs to initialization code common with the JPEG parser, the only difference being that this initialization code is invoked later in the ANI case, after some inputs have already been read from the input files. Regular dynamic symbolic execution also indicates that no regular input ever flows into the entire DLL containing this initialization code, although it cannot prove that no regular inputs are ever being cast into some untracked FP-value that later influences the execution of this initialization code. We nevertheless believe that it is unlikely that this code is ever called with attacker controllable inputs. A more sophisticated static and/or dynamic analysis would be needed to prove this automatically.

Although our static analysis declares $80 \%$ of all (FP-dependent and FP-independent) conditional jumps as being unsafe, it is good enough to reduce the number of runtime warnings about unsafe FP-dependent conditional statements to zero or two in the case of ANI. For the benchmarks considered, the algorithms of section 4 and 5 meet our initial goal of designing an 'as lightweight as possible' static analysis to prove non-interference between floating point code and security critical computations. 
Preliminary experiments with other Windows media parsers confirm the key observation made in this paper: for most programs in the application domain we consider, interactions between floatingpoint computations and memory allocation and indexing are extremely rare. For instance, parsing a sample small WMV (Windows Media Video) file with 27,401 bytes executes $252,715,214$ instructions (including 247,256,201 after the first input byte is read), but executes only 499 FP-instructions (i.e., less than $0.001 \%$ of the total number of instructions) and 29 FP-tainted conditional statements, which are proved attacker memory safe with our extended symbolic execution in 350 secs. We plan to optimize the memory usage of our current prototype implementation in order to handle longer execution traces and hence larger files and more complicated parsers.

\section{RELATED WORK AND DISCUSSION}

There are many other algorithms combining static and dynamic program analysis, some also aimed at proving memory safety [16] or type checking $[1,6]$. Our work can be viewed as following the same general strategy of "prove statically as much as possible, and use runtime checks as a fallback". However, a perhaps unique feature of our analysis is that the abstract domains used for the static and dynamic parts are quite distinct: we use a simple path-insensitive static analysis targeting FP instructions and tracking memory usage of individual if-then-else blocks, while we use a (bit-)precise dynamic symbolic execution to reason about the nonFP part of the program. The key novelty of our approach is the interplay between these two analyses and abstract domains, and the new notion of attacker memory safety that this combination is able to prove. Our analysis also attempts to prove a form of noninterference [18] between FP computations and memory accesses: FP values do not influence memory accesses.

In principle, the general strategy of over-approximating instructions not handled by symbolic execution could be used to prove memory safety in presence of instructions other than FP instructions, or to deal with any theory $\mathcal{T}^{\prime}$ of constraints outside $\mathcal{T}$ for which we do not want to generate constraints (because $\mathcal{T}^{\prime}$ is too complex, undecidable, expensive, or a solver is simply not readily available for whatever reason). However, this strategy seems to work well for FP instructions because of the expected non-interference of FP values (payload) with address computations (control). In practice, it is unclear if this strategy would work for other sets of unhandled instructions or constraints.

The static part of our analysis is conservative and handles multipointer dereferences (by returning 'unsafe'). However, the dynamic part as presented in Section 2 does not. To handle multiple levels of pointer dereferences and symbolic writes, the memory model used in Section 2 would need to be extended as discussed in [5] for instance. We did not consider this option here to simplify the exposition, as it is an orthogonal issue, and also because multipointer dereferences involving untrusted inputs are rare.

Recent work has started to address industrial-strength analysis of FP programs whose functional correctness is critical in some application domains like avionics [15]. Also, there is no doubt that SMT solvers will one day be extended to FP arithmetic. But for proving only memory safety of FP programs in the application domain we considered here, our work shows that precise FP reasoning is often not necessary, which is good news.

Obviously, blackbox fuzzing and other approaches to test generation based on coverage heuristics only are not limited by FP instructions. On the other hand, those approaches are less precise than whitebox fuzzing and can miss many bugs (for instance, all the security bugs found by SAGE during the development of Windows 7 [7] were actually missed by blackbox fuzzers and static program analysis). Moreover, blackbox approaches cannot prove memory safety, and fuzzing the payload part of an input that triggers a memory safe program execution is pointless, hence needlessly expensive.

Note that our technique is based on the idea that floating point code does not influence (attacker) memory safety. We specifically do not aim to prove correctness of the floating point computations. We also do not consider other application domains (from avionics, spec FP benchmarks, JavaScript programs where variables have floating-point value types by default, etc.) where the non-interference we seek may not hold. It is also trivial to write down toy programs for which our analysis technique would fail (simply cast a double to an integer and then index a buffer with that integer). The goal of our work is specifically to prove attacker memory safety of "nearly-secure" image and video parsers that are part of Windows and deployed on a billion PCs worldwide. Today, we are not aware of any other practical analysis and tool that can do this for the class and size of applications we consider.

\section{CONCLUSIONS}

In this paper, we introduced a new proof technique for attacker memory safety, which combines lightweight static analysis of floating-point parts of a program with a precise dynamic symbolic execution of the rest of the program.

We do not require theorem prover support for floating point, since our goal is not to reason precisely about the floating point logic of the program, but to prove attacker memory safety. Our intuition was verified in the examples we considered: the FP part does not interact in any dangerous way with buffer allocation or indexing.

As future work we plan to extend our combined analysis to cover the remaining cases of FP-dependent conditionals considered unsafe by our prototype. We also plan experiments with more file parsers, including more complex media players. Furthermore, since the combined proof strategy we propose is not generally linked to FP code, we will investigate other areas where dynamic test generation can benefit from replacing precise symbolic execution by a coarse and cheap over-approximation.

\section{REFERENCES}

[1] M. Abadi, L. Cardelli, B. Pierce, and G. Plotkin. Dynamic typing in a statically-typed language. In Conf. Rec. 16th Annu. ACM Symp. Principles of Programming Languages (POPL 1989), pages 213-227. ACM Press, Jan. 1989.

[2] G. Balakrishnan and T. W. Reps. Analyzing memory accesses in $\mathrm{x} 86$ executables. In 13th Int'l Conf. Compiler Construction (CC 2004), volume 2985 of LNCS, pages 5-23. Springer, Mar. 2004.

[3] C. Cadar, V. Ganesh, P. M. Pawlowski, D. L. Dill, and D. R. Engler. EXE: Automatically generating inputs of death. In ACM Conf. Computer and Communications Security (CCS 2006), pages 322-335. ACM Press, Oct. 2006.

[4] A. Edwards, A. Srivastava, and H. Vo. Vulcan: Binary transformation in a distributed environment. Technical Report MSR-TR-2001-50, Microsoft Research, 2001.

[5] B. Elkarablieh, P. Godefroid, and M. Levin. Precise pointer reasoning for dynamic test generation. In Proc. 18th Int'l Symp. Software Testing and Analysis (ISSTA'09), pages 129-139. ACM Press, July 2009.

[6] C. Flanagan. Hybrid type checking. In Proc. 33rd ACM SIGPLAN-SIGACT Symp. Principles of Programming Languages (POPL 2006). ACM Press, Jan. 2006. 
[7] P. Godefroid. Software model checking improving security of a billion computers. In Proc. 16th Int'l SPIN Workshop Model Checking Software (SPIN 2009), LNCS, page 1. Springer, June 2009.

[8] P. Godefroid, N. Klarlund, and K. Sen. DART: Directed automated random testing. In Proc. ACM SIGPLAN 2005 Conf. Programming Language Design and Implementation (PLDI 2005), pages 213-223. ACM Press, June 2005.

[9] P. Godefroid, M. Levin, and D. Molnar. Active property checking. In Proc. 8th ACM E IEEE Int'l Conf. Embedded software (EMSOFT'08), pages 207-216. ACM Press, Oct. 2008.

[10] P. Godefroid, M. Levin, and D. Molnar. Automated whitebox fuzz testing. In Proc. Network and Distributed System Security Symp. (NDSS 2008), pages 151-166. The Internet Society, Feb. 2008.

[11] Intel Corporation. Intel 64 and IA-32 Architectures Software Developer's Manual, 2009.

[12] J. Kinder and H. Veith. Jakstab: A static analysis platform for binaries. In 20th Int'l Conf. Computer Aided Verification (CAV 2008), volume 5123 of LNCS, pages 423-427. Springer, July 2008.

[13] T. Lengauer and R. Tarjan. A fast algorithm for finding dominators in a flowgraph. ACM Trans. Program. Lang. Syst., 1(1):121-141, 1979.
[14] D. Molnar, X. C. Li, and D. Wagner. Dynamic test generation to find integer bugs in $\mathrm{x} 86$ binary linux programs. In Proc. 18th USENIX Security Symp. USENIX Association, Aug. 2009.

[15] D. Monniaux. The pitfalls of verifying floating-point computations. ACM Trans. Program. Lang. Syst., 30(3):1-41, May 2008.

[16] G. C. Necula, S. McPeak, and W. Weimer. CCured: Type-safe retrofitting of legacy code. In Conf. Rec. POPL 2003: 29th SIGPLAN-SIGACT Symp. Principles of Programming Languages, pages 128-139. ACM Press, Jan. 2002.

[17] S. K. Raman, V. M. Pentkovski, and J. Keshava. Implementing streaming SIMD extensions on the Pentium III processor. IEEE Micro, 20(4):47-57, 2000.

[18] A. Sabelfeld and A. C. Myers. Language-based information-flow security. IEEE J. Sel. Areas Commun., 21(1):5-19, Jan. 2003.

[19] P. Wolper. Expressing interesting properties of programs in propositional temporal logic. In Conf. Rec. 13th Annu. ACM Symp. Principles of Programming Languages (POPL 1986), pages 184-192. ACM Press, Jan. 1986. 